\title{
A Proposal of Decentralised Architecture for Optimised Operations in Manufacturing Ecosystem Collaboration
}

\author{
Pavlos Eirinakis ${ }^{1}$, Jorge Buenabad-Chavez ${ }^{2}$, Rosanna Fornasiero ${ }^{3}$, Haluk Gokmen ${ }^{4}$, \\ Julien-Etienne Mascolo ${ }^{5}$, Ioannis Mourtos ${ }^{1}$, SvenSpieckermann ${ }^{6}$, Vasilis \\ Tountopoulos ${ }^{7}$, Frank Werner ${ }^{8}$ and RobertWoitsch ${ }^{9}$ \\ ${ }^{1}$ Athens University of Economics and Business, Greece, Peir@,Aueb.Gr, \\ ${ }^{2}$ University of Manchester, Uk, jorge@Manchester.Ac.Uk. \\ ${ }^{3}$ ITIA-CNR, Italy, rosanna.fornasiero@Itia.Cnr.It \\ ${ }^{4}$ Arcelik A.S., Turkey, Haluk.Gokmen@,Arcelik.Com \\ ${ }^{5}$ Centro Ricerche Fiat Scpa, Italy, Julienetienne.Mascolo@,Crf.It \\ ${ }^{6}$ Simplan Ag, Germany, Sven.Spieckermann@,Simplan.De \\ ${ }^{7}$ Athens Technology Center S.A., Greece, V.Tountopoulos@,Atc.Gr \\ ${ }^{8}$ Software AG, Germany, Frank.Werner@Softwareag.Com \\ ${ }^{9}$ BOC Asset Management Gmbh, Austria, Robert.Woitsch@boc-eu.com
}

\begin{abstract}
This paper describes an innovative approach to adopt the nextgeneration manufacturing paradigm based on flexible production units and ecosystems that can be quickly reprogrammed to provide fast time-to-market responses to global consumer demand, address mass-customisation needs and bring life to innovative products. The approach utilises the capabilities offered by digitalisation to facilitate (i) in-depth (self-) monitoring of machines and processes, (ii) decision support and decentralised (self-) adjustment of production, (iii) effective collaboration of the different IoT-connected machines with tools, services and actors (iv) seamless communication of information and decisions from and to the plant floor and (v) efficient interaction with value chain partners. The paper presents the conceptual architecture under development to support those functionalities for two specific domains in manufacturing.
\end{abstract}

Keywords: decentralized platforms, process optimization, simulation, multilayer platform.

\section{Introduction}

Industry 4.0 or the fourth industrial revolution [1] is the next developmental stage in the organisation and management of industrial processes and the entire manufacturing value chain. By blending the real and the virtual production world through digitalisation, companies will be able to connect all parts of the production process: machines, products, systems, and people. ICT-based systems and service platforms are already playing a major role in this transformation, enabling some forms of 
monitoring, analysis, simulation, optimization and control of production entities and processes. This is possible through the creation of a virtual copy of the physical world that facilitates decentralised structures of Cyber-Physical Systems (CPS)/Smart Objects (SO). Over the Internet-of-Things (IoT), CPSs communicate and cooperate with each other and humans in real-time. Via the Internet-of-Services (IoS), both internal and cross-organisational services are offered and utilised by participants of the value chain [2].

Such technologies have extensively been presented in the literature and have been the subject of various projects in application domains such as healthcare, energy grids and manufacturing. There are already related cloud offerings by Bosch for the smart office [3], and similar offerings by IBM [4], and Microsoft [5] among others.

The main contribution of the DISRUPT framework is a comprehensive solution to automation of vertical and horizontal operations and decision making for specific use cases in two project partner companies in the automotive and white goods sectors. We have already collected the requirements from these large companies and have developed the target use cases documentation. This paper presents the conceptual architecture to support these use cases. The architecture unifies previously separate production environments into a collaborative virtual ecosystem that seamlessly integrates cyber-physical operations, data analytics and decision support, while also incorporating the structural characteristics of the entire value chain. The architecture is currently being designed considering various reference architectures. We present its main modules in Section 3. Main design goals include: be a multi-sided, cloud-based platform that will enable those companies to reduce time, costs or resource consumption and to respond to unexpected events, fluctuations in consumer demand, massively customised products and global competition. It will facilitate the adoption of ICT-enabled innovation in manufacturing by vertically unifying the automation hierarchy of production systems, from IoT and sensors to ERP, MES and SCADA and from advanced analytics tools to production schedulers and capacity planners, all under a seamless data-intensive modelling approach. This will be implemented through modular, decentralised production topologies, empowering Smart Objects with analytics, simulation and optimisation tools and integrating them within a unified monitoring, control and decision support system, thus enabling them to act as intelligent autonomous agents inherent to the plant's virtual production model.

\section{Vision and Requirements}

In the traditional rigid factory, mass-production is the goal and cost-effectiveness is the drive; this has led many manufacturers to relocate to lower cost regions, and others to totally outsource their production. The new era of manufacturing asks for optimised plants and manufacturing chain networks, transforming them into profitable innovation centers. It requires flexible factories that can be quickly "reprogrammed" to provide faster time-to-market responding to global consumer demand, effectively addressing mass-customisation needs and bringing life to innovative new products. It needs transparent production processes that are responsive to changes or unexpected events originating throughout the value chain. It combines information, technology 
and human intellect, fundamentally changing how products are invented, manufactured, shipped and sold, materialising an interconnected, efficient manufacturing ecosystem. In that regard, the traditional automation pyramid with its layered, compartmentalised structure of automation systems seems unable to accommodate this transformation.

The proposed decentralized platform aims at disrupting the traditional automation pyramid (Fig. 1) by utilising the capabilities offered by modern ICT to facilitate (i) indepth (self-) monitoring of machines and processes, (ii) decision support and decentralised (self-)adjustment of production, (iii) effective collaboration of the different IoT-connected machines and devices with tools, services and actors (iv)seamless communication of information, knowledge, and decisions from and to the plant floor and (v) efficient interaction with value chain partners [2].

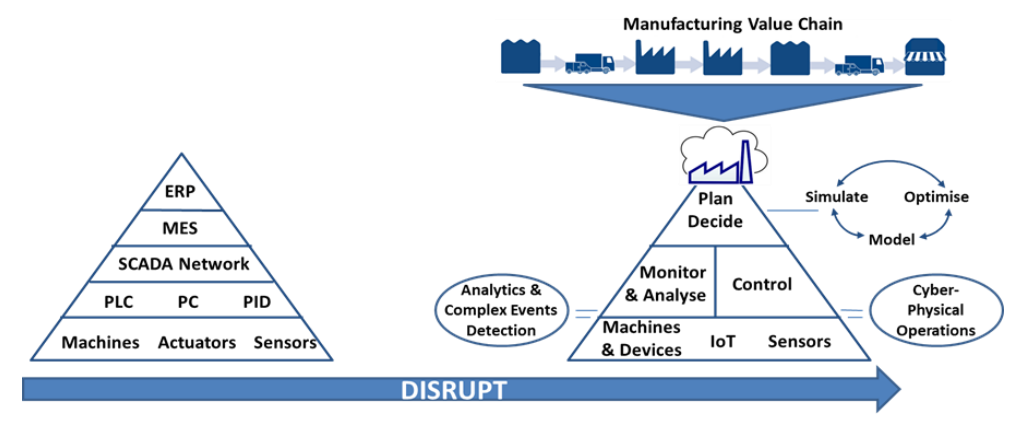

Fig.1: The DISRUPT Concept: Disrupting the traditional automation pyramid

More specifically, within DISRUPT, each physical element of production (machine, device, sensor, etc.) is monitored and controlled via the IoT by its virtual counterpart. The vast amount of data collected is processed, refined into information and analysed to detect complex events that, in turn, trigger actions fed back to the physical production units or presented to decision makers along with the appropriate tools to handle them. To that end, DISRUPT proposes a set of decision support tools based on three core interrelated and interacting modules: modelling, simulation and optimisation. These tools should not only incorporate a detailed representation of the production process and the plant floor but also correlate the manufacturing process with information derived from the whole value chain in real-time, refined and analyzed by streaming and predictive analytics as well as complex event processing tools. The platform is cloud-based to accommodate the anticipated high data volume and the computational needs that this volume implies, thus enabling scalability.

The DISRUPT approach is generic to cover different manufacturing settings and scenarios. Nevertheless, it is driven by three concrete business cases that derive from the automotive and the white goods sector, namely:

Market-driven production reconfiguration, scaling and optimisation: Rapid product lifecycles and mass customisation imply an increasing number of phases in product deployment and the involvement of actors along the value chain. Hence, it becomes even more important to consistently reconfigure production processes, to 
achieve reduced costs and time-to-market, smaller lot sizes and production volumes. Indicatively, this business case is highly applicable to the white goods sector.

Ecosystem-aware, event-enabled production planning and control: This stems from the need to ensure business continuity in the ever-changing contemporary manufacturing environment, where production goals are often derailed by late-cycle changes, the use of unqualified and nonstandard parts, unexpected plant floor events, low supplier involvement and the lack of proper decision support tools to handle the above. Indicatively, this case applies to the automotive sector.

Automated plant floor event handling and self-adjustment: The notion of CPS lies at the very core of Industry 4.0. The incorporation of IoT as a core component of CPSs and their inherent large-scale nature raise a number of specific challenges ranging from system-level management and control to data analytics. On the same time, the evolution of CPSs bundled with analytics and decision support tools leads to manufacturing components that incorporate self-adjustment capabilities.

\section{Conceptual Architecture and Modules}

The under development reference platform is designed to allow the incorporation of a variety of different tools and services and facilitates a wide range of manufacturing activities from day-to-day and responsive operations to strategic planning, combining an in-depth approach to the actual manufacturing process and the plant floor with a macroscopic value chain perspective. The platform is based on a distributed ServiceOriented-Architecture (SOA) that interconnects the various modules in a seamless manner and provides a "plug-n-play" feel for services and tools to be incorporated in a fast, easy and straightforward manner. Semantic interoperability is utilised to allow the harmonious collaboration of its different modules, enabling them to interact exchanging data with unambiguous, shared meaning. In that regard, the model of the actual manufacturing unit and its value chain relations needs to be conceptually represented via appropriate ontologies of materials, resources, actors, products, machines and processes.

Fig. 2 provides a schematic representation of the proposed architecture. The system enables interaction with the actual plant floor (via CPSs and the IoT interrelating machines, sensors, systems and devices), with the manufacturing organisation's Enterprise Information Systems and its Ecosystem (i.e., its value chain partners as well as software developers). 


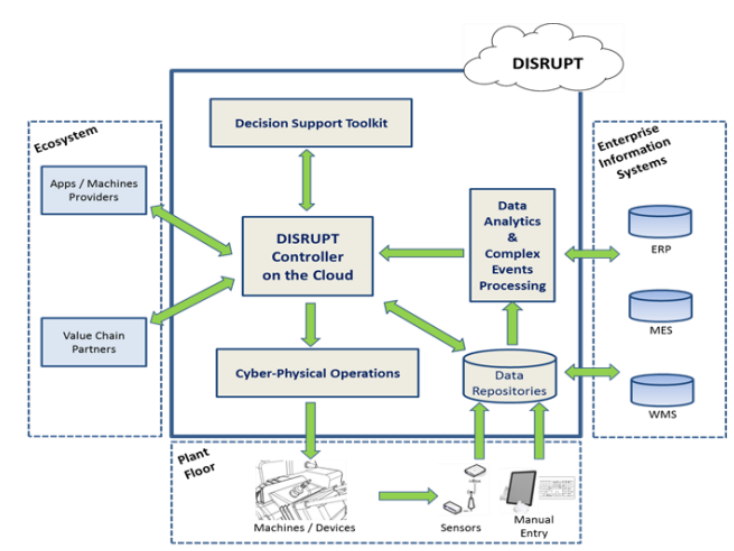

Fig.2. The DISRUPT System Architecture

The DISRUPT system architecture is structured in four interrelated modules.

Module 1: Data Analytics and Complex Event Processing. This module collects, analyses and handles data from diverse sources like specialized sensors or metering devices located at the plant floor along the production process interconnected via IoT, systems embedded in production machinery, on-site or asynchronous manual data entry and Enterprise Information Systems (e.g., ERP, MES, WMS). Depending on their source and form, the appropriate data are retrieved and filtered to avoid transmitting duplicate or non-useful data and processed (if needed) to attain their desirable form. Moreover, this module analyses the vast amount of data pouring in, turning them into meaningful real-time metrics. It detects and analyses patterns, thus producing valuable production, efficiency and business knowledge, addressing "what happened" (querying \& reporting), "why it happened" (analysis) and "what will happen" (predictive \& streaming analytics).

By utilizing Complex Event Processing (CEP), it allows manufacturing organizations to immediately respond with timely and relevant actions related to operational activities by correlating and analysing events across multiple data streams in real-time. Furthermore, it enables intelligent automated action on fast-moving big data, triggering low-latency actions automatically without human input. To that end, this module will filter, aggregate, enrich and analyse a high throughput of data from multiple disparate live data sources in any data format to identify simple and complex patterns, detect urgent situations and automate immediate response. The integration of sophisticated analytics with native support for temporal arguments and offer flexible event replay for testing new event scenarios and analysing existing ones.

The architectural approach for this module (Fig.3) is event-driven, collecting information from different sources in vertical and horizontal networks, supporting a plethora of scenarios and simultaneous processing of discrete and streaming events. This architecture guarantees load-balancing and scalability and supports IoT clients with a single messaging platform. To handle the available vast amount of data, it will be empowered by cache static data for fast, in-memory access. 


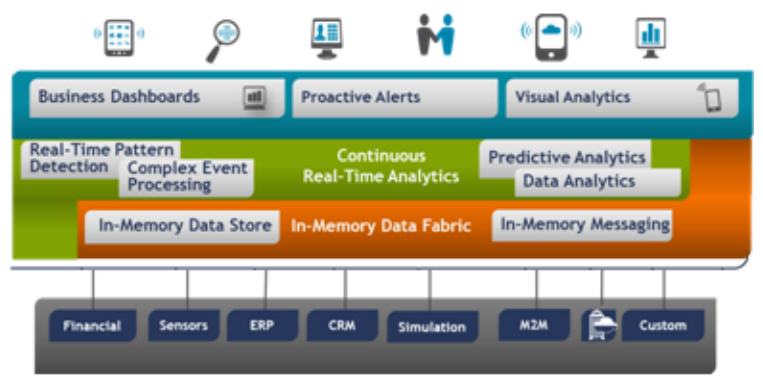

Fig.3. The DISRUPT Streaming Analytics Architectural Approach

Module 2: Cyber-Physical Operations. This module handles the interaction with the production level via the organization's manufacturing systems and enables selfadjustment and self-configuration via a network of modular, decentralized Smart Objects, offering synchronization between the physical plant floor and its virtual representation. More specifically, it accommodates the control of factory operations at machine level by integrating the dynamics of the physical processes with those of software and networking, providing abstractions and tools for each component separately as well as for the integrated whole. To that end, the new platform will adopt a modular, flexible CPS structural architecture that empowers production schemes by facilitating both robustness and concurrent development, offering seamless integration of the organization's CPSs and the simple evolution and incorporation of other existing systems. Modularity does not only serve the purpose of providing a framework for easily integrating CPSs. It also accommodates and empowers the actual operations of such systems. More specifically, the platform will include a virtual counterpart (Smart Object-SO) for each physical entity that not only represents the entity in the virtual factory but also provides computing and networking capabilities to the actual machine. These SOs will be bundled in networks according to the model of the corresponding process step, which will be subsequently interrelated with other process steps to form the processes that comprise the production scheme. Hence, the entire production will be virtualised as a structure of decentralized systems of cooperating, autonomous Smart Objects (Fig.4) based on the 5C level architecture for CPS[6],[7].

The adopted 5C level architecture will enable DISRUPT CPSs to process and analyse information and handle events at the appropriate level, having the visibility that is required to address the corresponding issue, spanning from local to crossprocess or global. Note that with respect to the DISRUPT project this architecture cannot be supported via only local deployment. A cloud-based approach must be followed to deal with the vast amount of data and the associated processing power. However, in order to facilitate the adoption of existing CPSs, DISRUPT will also allow for hybrid deployment configurations, including SOs that perform part of the processing activities locally (e.g., perform conversion level analytics locally, at individual sensor nodes, while performing cyber-level methods on the cloud). 


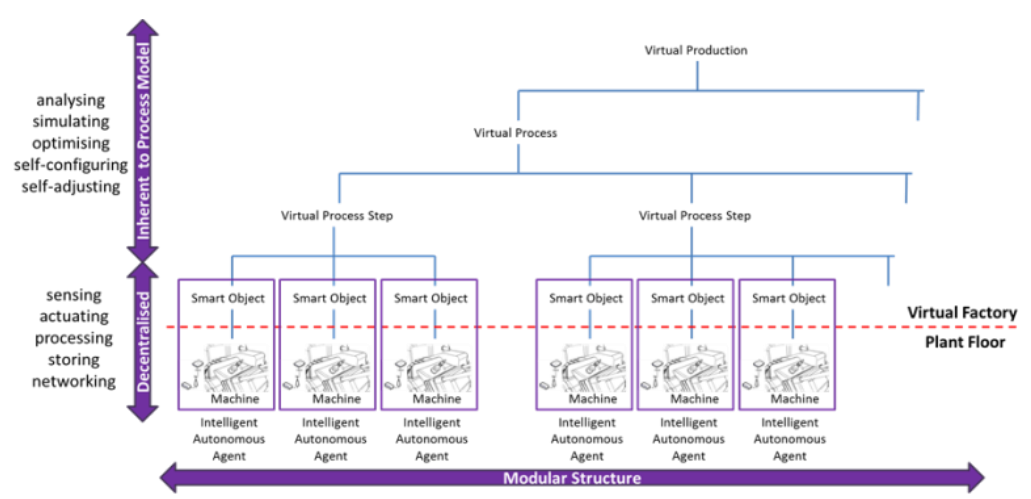

Fig.4. The DISRUPT Modular, Decentralised CPS Structure

Module 3: the decision support toolkit. This module consists of 3 interrelated sub-modules, i.e., Modeling, Simulation and Optimisation, that interact to offer a wide range of decision support tools that cover multiple aspects of the production process or the entire manufacturing chain. Some indicative tools provided by this module are as follows.

Modelling and Design tool: Enterprise Modelling is a particular field in Conceptual Modelling that describes an enterprise in a holistic way in order to enable model processing. In Enterprise Modelling, the business (domain specific aspects) and ICT (technical aspects) are aligned with the organisational goals. Considering a manufacturing chain as a virtual chain that involves multiple independent enterprises, institutions and people which come together to achieve a certain goal [8], [9], the approach of enterprise modelling can be adapted in the context of Industry 4.0 in order to holistically describe the manufacturing processes as well as the technical production level of the whole manufacturing chain. The domain-specific business aspects are the embedded production processes, whereas the technical aspects are the production lines with analogies to enterprise workflows that distinguish between manual, semi-automatic and automatic processes. Moreover, Production Case Management is utilized to enable introducing the flexibility of production processes in a holistic modelling approach independently from the usage of IDEF0, BPMN, or any other modelling method.

Co-Simulator environment: Co-simulation allows coupling simulation environments at runtime in order to link different model descriptions, different parts/components of the overall systems, and possibly different computational algorithms [10]. The communication, the data exchange and the data synchronization is coordinated via a middleware assuming in most cases fixed lengths of communication intervals and a common modelling approach (also denoted as a co-model [11]). The main tasks that should be fulfilled for the development of a decentralised, self-organising cosimulation framework is the communication setup, the control of the co-simulation procedure, the synchronisation of the data exchange and the extrapolation strategies. Moreover, the co-simulation framework must allow its integration with optimisation and predictive analytics to allow not only effective automation in individual plant floors but also for the intensive collaboration of manufacturing sites. 
Production Optimisation: This module will be based on algorithms to solve complex scheduling problems with various side constraints, such as utility (renewable resources) and machine maintenance constraints, to offer the capability of treating multiple scheduling objectives in a hierarchical fashion, including combined temporal and energy-aware objectives [12], [13]. In particular, the platform will develop scheduling algorithms in several ways that go beyond the current state-of-the-art, adding significantly to the provided level of accuracy and enhancing their planning capability as follows: i) Multiple machine configurations will be incorporated into the existing models as decision variables. The available configurations control the operating conditions (such as the speed and the temperature), and they affect not only the completion time but also the energy consumption and the operating cost; ii) The level of work-in-progress (WIP) inventory that is kept in front of machines (or among the process steps) as well as lot-sizing variables will be considered; iii) Multiple execution modes will be added, including among others alternative routings and combinations of resource requirements for each production order or operation. For this purpose, in addition to the utility constraints based on renewable resources, nonrenewable and doubly constrained resources will be considered. Non-renewable resources are limited for all periods and the entire scheduling horizon, while doubly constrained resources are limited both period by period and for the entire scheduling horizon [14].

Module 4. Controller on the Cloud. This module integrates all tools and modules, enabling their effective collaboration and the seamless communication of information, knowledge, and decisions to the plant floor and across the value chain. The Controller is based upon an integrated meta-model of the major factory modules. In order to realize its full potential, three aspects will be implemented within DISRUPT [15]: horizontal integration across the manufacturing organization, vertical integration of different production modules and SOs, and cross-boundary integration to cover the products' full lifespan.

Events detected by CEP will trigger the system towards possible changes in the Decision Support Toolkit (e.g., involving changes in model design, testing their effects through simulation, utilizing the appropriate optimisation tools) and possibly in the Cyber-Physical Operations module, communicating the appropriate system response to the plant-floor. Such response may be human-controlled or automatic, an example being a machine predicting failures and triggering maintenance processes autonomously. Similarly, changes in production because of exogenous reasons (e.g., involving actors in the value chain) will be communicated by the Controller to the Decision Support Toolkit, hence allowing for real-time production re-scheduling that will be subsequently fed into production.

The Controller also offers an Ecosystem Gateway. The role of this gateway is twofold. Firstly, it offers a reliable and secure interface for the collaboration of the manufacturing unit with value chain partners, through which all related actors and modules are able to communicate changes in real-time thus virtualizing the actual setting under which the factory's production is implemented. This may include changes in orders by the customers, daily directives from centralized management, the introduction of new partners or unexpected events. Secondly, it allows cooperation with software developers, technology and machine providers, offering a standardized framework for them to interact with DISRUPT. It provides them with the necessary 
tools for adopting and utilizing the production and value chain semantics scheme, recalibrate their products and offer ready-to-deploy solutions, thus enabling them to showcase the business value of their tools/services/machine utilizing a channel where they can be properly pre-tested and evaluated.

\section{Conclusions}

The decentralised architecture presented is part of the DISRUPT project [15]. Its purpose is to fully automate vertical and horizontal operations and decision making in project partner companies in the automotive and white goods sectors. We have presented its conceptual architecture and described the functionality of its main modules.

We are currently designing the software architecture details based on already gathered requirements and modelling of specific use cases for each of those sectors; we are also considering the reference architectures by the Industrial Internet Consortium (IIC) [16], the German initiative Industrie 4.0 [17], the EU project IoT-A Internet of Things Architecture [18], and the work-in-progress ISO/IEC Internet of Things Reference Architecture [19]. These reference architectures provide guidelines for the design and development of IoT systems based on standards in order to ensure interoperability.

Many of the advances proposed for the DISRUPT architecture are already used in manufacturing and have been the subject of the literature extensively and similar projects around the world. The ambition of the DISRUPT approach is to radically transform manufacturing in those specific sectors and use cases, through the harmonious integration of those advances into a holistic manufacturing system: isolated, optimised cells that come together as a fully integrated, automated, and optimised production flow, leading to greater efficiencies and changing traditional production relationships among suppliers, producers and customers, as well as between humans and machines.

Our aim is for the DISRUPT platform to be a proof-of-concept in, and a reference implementation for, the automotive and white goods sectors. As a comprehensive solution, it should also be of use in other application domains.

Acknowledgments. The work on this paper is funded mainly by the European Commission through the DISRUPT project (H2020 FOF-11-2016, RIA project n. 723541, 2016-2018). The authors would also like to thank the contributions of the different partners of the DISRUPT project.

\section{References}

[1] Kagermann, H., Wahlster, W., and J. Helbig, eds. Recommendations for implementing the strategic initiative Industrie 4.0: Final report of the Industrie 4.0 Working Group, 2013. 
[2] Hermann, M., Pentek, T., and B. Otto (2015). Design Principles for Industrie 4.0 Scenarios. Working Paper No. 01 / 2015.

[3] Bosch IoT Platform: https://www.bosch-si.com/iot-platform/bosch-iot-suite/homepagebosch-iot-suite.html

[4] IBM Watson Internet of Things: https://www.ibm.com/internet-of-things/

[5] Microsoft Internet of Things: https://www.microsoft.com/en-gb/internet-of-things/

[6] Lee, J., Bagheri, B., and H.-A. Kao (2015). A Cyber-Physical Systems architecture for Industry 4.0-based manufacturing systems. Manufacturing Letters 3, 18-23.

[7] Bagheri, B. and J. Lee. Big future for cyber-physical manufacturing systems. Design World (September 23, 2015) http://www.designworldonline.com/big-future-for-cyberphysical-manufacturing-systems/

[8] Arnold, D., Faisst, W., Haertling, M. and P. Sieber (1995). Virtuelle Unternehmenals Unternehmenstyp der Zukunft? HMD Theorie und Praxis der Wirtschaftsinformatik 32 (185).

[9] Kanet, J.J., Faisst, W., and P. Mertens (1999). Application of information technology to a virtual enterprise broker: The case of Bill Epstein. International Journal of Production Economics, 62, 23-32

[10] Bleicher F., Duer F., Leobner I., Kovacic I., Heinzl B., and W. Kastner (2014). Cosimulation environment for optimising energy efficiency inproduction systems. CIRP Annals - Manufacturing Technology 63, 441-444.

[11] J. Fitzgerald et al. (eds.), Collaborative Design for Embedded Systems, Springer-Verlag Berlin Heidelberg 2014.

[12] Plitsos S., Repoussis P.P., Mourtos I., and C.D. Tarantilis (2015). Energy-aware decision support for production scheduling, Decision Support Systems (under review).

[13] Repoussis P.P., Paraskevopoulos D.C. and C.D. Tarantilis (2015). Iterated Local Search Algorithm for Flexible Job Shop Scheduling Problems with Resource Constraints. POMS 2015 Annual Conference, May 8-11, Washington, USA.

[14] Alcaraz J. Maroto C. and R. Ruiz (2003). Solving the Multi-Mode Resource-Constraint Project Scheduling Problem with Genetic Algorithms, Journal of the Operational Research Society 54, 614-626.

[15] http://www.disrupt-project.eu/

[16] Industrial Internet Consortium Reference Archtitecture: https://www.iiconsortium.org/IIRA-1-7-ajs.pdf

[17] Reference Architecture Model Industrie 4.0: https://webstore.iec.ch/preview/info_iecpas63088\%7Bed1.0\%7Den.pdf

[18] IoT-A Internet of Things Architecture Project Final Report: http://cordis.europa.eu/docs/projects/cnect/1/257521/080/reports/001257521IoTAPFRrenditionDownload.pdf

[19] ISO/IEC CD 30141:20160910(E). Internet of Things Reference Architecture (IoT-RA): https://www.w3.org/WoT/IG/wiki/images/9/9a/10N0536 CD text of ISO IEC 30141.p $\underline{\text { df }}$ 